\title{
Differences in coronary plaque characteristics between patients with and those without peripheral arterial disease Krzysztof L. Bryniarski ${ }^{a}$, Erika Yamamoto ${ }^{a}$, Higuma Takumi ${ }^{a}$, Lei Xing ${ }^{a}$, Thomas Zanchin ${ }^{a}$, Tomoyo Sugiyama ${ }^{a}$, Hang Lee ${ }^{b}$ and Ik-Kyung Jang ${ }^{a, c}$
}

\begin{abstract}
Introduction Cardiovascular mortality of patients with combined peripheral arterial disease (PAD) and coronary artery disease (CAD) is twice as high as that in those with either disease alone. It is known that patients with PAD undergoing percutaneous coronary intervention have a higher incidence of adverse cardiac events such as myocardial infarction or target vessel revascularization.
\end{abstract}

Objective In this study, we compared the detailed characteristics of culprit and nonculprit plaques between patients with and those without PAD using optical coherence tomography.

Patients and methods We performed propensity score matching using the following variables: (i) age; (ii) sex; (iii) clinical presentation; (iv) diabetes mellitus; (v) hyperlipidemia; (vi) smoking; (vii) hypertension; (viii) BMI; and (ix) coronary lesion location. Finally, we matched 34 culprit lesions and 30 nonculprit lesions in patients with PAD to 68 culprit lesions and 60 nonculprit lesions in patients without PAD (1: 2 ratio).

Results In culprit lesions, PAD patients when compared with those without PAD had a higher prevalence of lipid-rich plaque (73.5 vs. $51.5 \% ; P=0.033$ ), higher lipid index (1744 \pm 1110 vs. $1246 \pm 656 ; P=0.043)$, calcification

\section{Introduction}

The prevalence of peripheral artery disease (PAD) increases with age, reaching up to $20 \%$ in patients older than 70 years [1]. PAD is associated with both higher clinical event rates and mortality [2]. Most of these clinical events are attributed to coronary atherosclerosis the cardiovascular risk profile is more pronounced in concomitant coronary artery disease (CAD) and PAD [3-5]. In the REACH registry, the 1-year risk for cardiovascular death, myocardial infarction, stroke, or hospitalization for atherothrombotic events exceeded 23\% in patients with concomitant $\mathrm{PAD}$ and $\mathrm{CAD}$, whereas it ranged between 13 and $17 \%$ for either disease alone. All-cause mortality of patients with combined lowerextremity PAD and CAD was twice as high as that in those with either disease alone [4]. Further, patients with PAD undergoing percutaneous coronary intervention are

Supplemental digital content is available for this article. Direct URL citations appear in the printed text and are provided in the HTML and PDF versions of this article on the journal's website, www.coronary-artery.com.

0954-6928 Copyright (c) 2017 Wolters Kluwer Health, Inc. All rights reserved.
(79.4 vs. $58.8 \% ; P=0.039$ ), macrophage accumulation (70.6 vs. $48.5 \% ; P=0.034$ ), and cholesterol crystals ( 32.4 vs. 10.3\%; $P=0.006)$. In nonculprit lesions, PAD patients had a higher prevalence of calcification (76.7 vs. $55.0 \% ; P=0.046$ ), macrophage accumulation (63.3 vs. $38.3 \% ; P=0.025)$, and cholesterol crystals ( 36.7 vs. $16.7 \% ; P=0.034$ ).

Conclusion Our study suggests greater coronary plaque vulnerability in both culprit and nonculprit lesions in patients with PAD. This observation underscores the need for more aggressive risk management in patients with combined PAD and coronary artery disease. Coron Artery Dis 28:658-663 Copyright (C) 2017 Wolters Kluwer Health, Inc. All rights reserved.

Coronary Artery Disease 2017, 28:658-663

Keywords: coronary artery, inflammation, intravascular imaging, optical coherence tomography, plaque vulnerability

${ }^{a}$ Cardiology Division, ${ }^{b}$ Biostatistics Center, Massachusetts General Hospital, Harvard Medical School, Boston, Massachusetts, USA and ${ }^{\mathrm{C} D i v i s i o n}$ of Cardiology, Kyung Hee University Hospital, Seoul, Republic of Korea

Correspondence to Ik-Kyung Jang, MD, PhD, Cardiology Division, Massachusetts General Hospital, Harvard Medical School, GRB 800, 55 Fruit Street, Boston, MA 02114, USA

Tel: +1 617726 9226; fax: +1 617726 7416; e-mail: ijang@mgh.harvard.edu

Received 2 May 2017 Revised 12 June 2017 Accepted 14 June 2017

known to have a higher incidence of adverse cardiac events such as myocardial infarction or target vessel revascularization [6]. Differences in coronary plaque phenotype may explain the differences in outcomes. Therefore, in this study, we compared the detailed characteristics of both culprit and nonculprit plaques between patients with and those without PAD using optical coherence tomography (OCT).

\section{Patients and methods}

\section{Study population}

Participants were selected from the Massachusetts General Hospital (MGH) OCT Registry. The MGH OCT Registry is an ongoing, international, multicenter registry of patients who have undergone OCT of the coronary arteries and involves 20 sites across six countries (NCT01110538). In our study, PAD was defined as follows: previous aorto-femoral bypass surgery, limb bypass surgery, or percutaneous transluminal angioplasty of the iliac or infrainguinal arteries; previous limb or foot 
amputation for arterial vascular disease; carotid artery stenosis, significant peripheral artery stenosis; or ankle/ arm blood pressure ratio less than 0.80 [7]. We have identified 88 patients with PAD and performed propensity score matching between lesions of patients with and lesions of those without PAD at a $1: 2$ ratio. As previous studies have reported that (i) age, (ii) sex, (iii) clinical presentation, (iv) diabetes mellitus, (v) hyperlipidemia, (vi) smoking, (vii) hypertension, and (viii) BMI influence plaque burden and plaque characteristics, we have used those variables as matching criteria [8-11]. In addition, we have matched for coronary lesion location. As a patient has only one culprit lesion, patient or lesion can be used interchangeably. However, with regard to nonculprit lesion, one patient may have two or more nonculprit lesions in different segments or even in different vessels. Therefore, nonculprit lesion of each PAD patient was matched to two lesions in the same segment of the artery in non-PAD patients. Even though we matched lesion locations, the lesion phenotype was not matched. Patients without prepercutaneous coronary intervention OCT, with poor image quality of OCT, or with incomplete imaging of the entire lesion were excluded from the analysis $(n=44)$. The registry was approved by the Institutional Review Board at each participating site. Written informed consent was obtained from all patients before enrollment.

\section{Image acquisition and analysis}

A frequency-domain OCT system (St. Jude Medical, St. Paul, Minnesota, USA) and a time-domain OCT system (LightLab Imaging Inc., Westford, Massachusetts, USA) were used to acquire images. The technique of intracoronary OCT imaging has been previously described [12]. All images were de-identified, digitally stored, and submitted to the Massachusetts General Hospital (Boston, Massachusetts, USA). All OCT images were analyzed at every 1 -mm interval.

Lipid-rich plaque (LRP) was defined as a plaque with maximum lipid arc greater than $90^{\circ}$. Lipid index was calculated as mean lipid arc multiplied by lipid length [13]. In addition, the presence or absence of thin-cap fibroatheroma (TCFA), macrophage accumulation, cholesterol crystal, and microvessel was noted. TCFA was defined as a LRP with fibrous cap thickness less than or equal to $65 \mu \mathrm{m}$ [14]. Macrophage accumulation on the OCT images was identified by increased granular signal intensity within the plaque accompanied by heterogenous back shadows $[15,16]$. Cholesterol crystals were characterized as high-intensity thin linear region adjacent to a lipid core. Microvessel was defined as small vesicular or tubular structures with diameter $50-300 \mu \mathrm{m}$. Calcification was also recorded in areas with low backscatter and sharp borders inside a plaque [17].

\section{Statistical analysis}

Categorical data are presented as counts and \%. The normality of distributions of continuous variables was examined using the Kolgormonov-Smirnov test. The mean and SD are reported for normally distributed data, and the median (25th-75th percentiles) is reported for data that were not normally distributed. Fisher's exact test or the $\chi^{2}$-test was used to analyze categorical variables and Student's $t$-test or Mann-Whitney's $U$-test for continuous variables. Statistical significance was defined as $P$ less than 0.05 . All statistical analyses were performed with SPSS 23.0 (SPSS Inc., Chicago, Illinois, USA).

\section{Results \\ Culprit lesions}

We matched 34 culprit lesions of 34 PAD patients to 68 lesions of 68 patients without PAD. The average age was 65 years and $74(72.5 \%)$ patients were male (Table 1$)$. $\beta$-Blockers were less frequently used in PAD patients and approximately one-third of the patients presented with stable angina.

With regard to culprit plaques, PAD patients had a higher prevalence of LRP with greater lipid burden, calcification, macrophage accumulation, and cholesterol crystals (Fig. 1 and Table 2). There was a trend toward higher prevalence of TCFA in the PAD group.

\section{Nonculprit lesions}

We matched 30 nonculprit lesions from 21 PAD patients to 60 nonculprit lesions from 56 patients without PAD. In the subgroup of patients with nonculprit lesions (demographics in Supplementary Tables, Supplemental digital content 1, http://links.lww.com/MCA/A157 and Supplemental digital content 2, http://links.lww.com/MCA/A158) PAD patients had longer plaques and higher prevalence of calcification,

Table 1 Baseline characteristics

\begin{tabular}{lccc}
\hline & PAD $(n=34)$ & Non-PAD $(n=68)$ & $P$ \\
\hline Age (mean \pm SD) (years) & $65.4 \pm 10.2$ & $64.6 \pm 4.3$ & 0.680 \\
Sex (male) & $24(70.6)$ & $50(73.5)$ & 0.754 \\
BMI (mean \pm SD) $\left(\mathrm{kg} / \mathrm{m}^{2}\right)$ & $25.3 \pm 3.9$ & $26.0 \pm 4.3$ & 0.405 \\
Diabetes mellitus & $16(47.1)$ & $26(38.2)$ & 0.393 \\
Hypertension & $24(70.6)$ & $50(73.5)$ & 0.754 \\
Hyperlipidemia & $27(79.4)$ & $51(75.0)$ & 0.620 \\
Current smoker & $11(32.4)$ & $20(29.4)$ & 0.761 \\
Medications & & & \\
$\quad$ Aspirin & $21(61.8)$ & $40(58.8)$ & 0.775 \\
Clopidogrel & $9(26.5)$ & $12(17.6)$ & 0.299 \\
$\quad$-Blocker & $6(17.6)$ & $26(38.2)$ & 0.035 \\
$\quad$ ACEI or ARB & $12(35.3)$ & $25(36.8)$ & 0.884 \\
$\quad$ Statin & $23(67.6)$ & $34(50.0)$ & 0.091 \\
Presentation & & & 0.627 \\
$\quad$ Stable angina & $11(32.4)$ & $22(32.4)$ & \\
Unstable angina & $15(44.1)$ & $29(42.6)$ & \\
$\quad$ AMl & $8(23.5)$ & $17(25.0)$ & \\
\hline
\end{tabular}

Values are mean \pm SD or $n(\%)$

ACEI, angiotensin-converting enzyme inhibitor; AMI, acute myocardial infarction; $\mathrm{ARB}$, angiotensin II receptor blocker; PAD, peripheral artery disease.

Bold values statistically significant $(P<0.05)$. 
Fig. 1
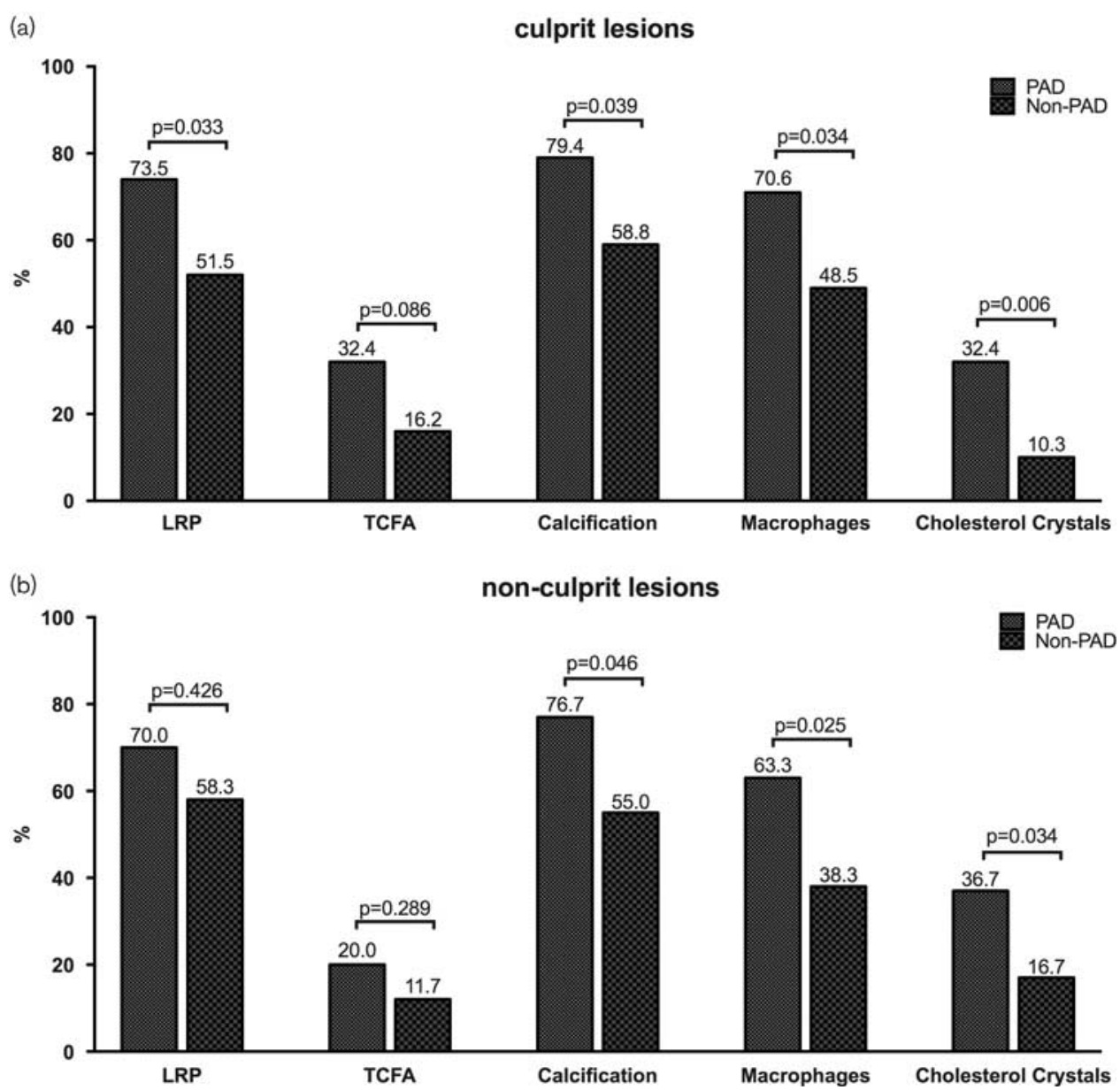

Comparison of culprit and nonculprit plaque characteristics between patients with and those without peripheral artery disease. (a) Culprit lesions in patients with peripheral artery disease (PAD) had significantly higher prevalence of lipid-rich plaque (LRP), calcification, macrophages, and cholesterol crystals. (b) Although there were no differences in the prevalence of LRP in nonculprit lesions between patients with and those without PAD, we observed significant differences in the frequency of calcification, macrophages and cholesterol crystals. Thin-cap fibroatheroma (TCFA) was twice as frequent in both culprit and nonculprit lesions of patients with PAD as compared with that in patients without PAD (a,b).

Table 2 Culprit lesions optical coherence tomography findings

\begin{tabular}{lccc}
\hline & PAD $(n=34)$ & Non-PAD $(n=68)$ & $P$ \\
\hline Plaque length (mm) & $16.8 \pm 7.7$ & $16.4 \pm 6.6$ & 0.844 \\
Lipid-rich plaque & $25(73.5)$ & $35(51.5)$ & $\mathbf{0 . 0 3 3}$ \\
$\quad$ Lipid core length (mm) & $10.6 \pm 5.2$ & $8.9 \pm 3.9$ & 0.158 \\
Mean lipid arc (deg.) & $171.1 \pm 35.3$ & $140.5 \pm 30.8$ & $\mathbf{0 . 0 0 1}$ \\
Max lipid arc (deg.) & $257.4 \pm 81.0$ & $206.3 \pm 65.1$ & $\mathbf{0 . 0 0 2}$ \\
Lipid index & $1744 \pm 1110$ & $1246 \pm 656$ & $\mathbf{0 . 0 4 3}$ \\
TCFA & $11(32.4)$ & $11(16.2)$ & 0.086 \\
FCT (mm) & $0.08 \pm 0.04$ & $0.10 \pm 0.04$ & 0.112 \\
Calcification & $27(79.4)$ & $40(58.8)$ & $\mathbf{0 . 0 3 9}$ \\
Macrophage accumulation & $24(70.6)$ & $33(48.5)$ & $\mathbf{0 . 0 3 4}$ \\
Microvessel & $11(32.4)$ & $17(25.0)$ & 0.433 \\
Cholesterol crystals & $11(32.4)$ & $7(10.3)$ & $\mathbf{0 . 0 0 6}$ \\
\hline
\end{tabular}

Values are mean $\pm \mathrm{SD}$ or $n(\%)$.

FCT, fibrous cap thickness; PAD, peripheral artery disease; TCFA, thin-cap fibroatheroma.

Bold values statistically significant $(P<0.05)$.
Table 3 Nonculprit lesions optical coherence tomography findings

\begin{tabular}{lccr}
\hline & PAD $(n=30)$ & Non-PAD $(n=60)$ & $P$ \\
\hline Plaque length (mm) & $15.6 \pm 5.5$ & $10.5 \pm 4.6$ & $<0.001$ \\
Lipid-rich plaque & $21(70.0)$ & $35(58.3)$ & 0.426 \\
$\quad$ Lipid core length (mm) & $8.0 \pm 2.8$ & $6.9 \pm 3.0$ & 0.180 \\
Mean lipid arc (deg.) & $139.7 \pm 20.2$ & $133.8 \pm 22.4$ & 0.328 \\
Max lipid arc (deg.) & $173.4 \pm 32.1$ & $166.7 \pm 48.1$ & 0.574 \\
Lipid index & $1091 \pm 393$ & $929 \pm 475$ & 0.194 \\
TCFA & $6(20.0)$ & $7(11.7)$ & 0.289 \\
FCT (mm) & $0.09 \pm 0.03$ & $0.10 \pm 0.03$ & 0.437 \\
Calcification & $23(76.7)$ & $33(55.0)$ & 0.046 \\
Macrophage accumulation & $19(63.3)$ & $23(38.3)$ & 0.025 \\
Microvessels & $13(43.3)$ & $29(48.3)$ & 0.654 \\
Cholesterol crystals & $11(36.7)$ & $10(16.7)$ & 0.034 \\
\hline
\end{tabular}

Values are mean $\pm \mathrm{SD}$ or $n(\%)$.

FCT, fibrous cap thickness; PAD, peripheral artery disease; TCFA, thin-cap fibroatheroma.

Bold values statistically significant $(P<0.05)$. 
macrophage accumulation, and cholesterol crystals (Fig. 1 and Table 3).

\section{Discussion}

Patients with PAD are known to have greater plaque burden, inflammatory burden, and worse endothelial function, which may explain higher cardiovascular events [18]. The present study provides insight into the coronary plaque characteristics of patients with concomitant PAD and CAD. In our study, we showed that culprit lesions of PAD patients compared with those in patients without PAD had greater lipid content as well as more features of vulnerable plaque, including higher prevalence of macrophages. Likewise, nonculprit lesions in PAD patients had more features of plaque vulnerability.

\section{OCT findings}

In an intravascular ultrasound study, Hussein et al. [19] demonstrated that patients with both CAD and PAD had greater plaque burden in the coronary trees. However, intravascular ultrasound with its low axial resolution (150-200 $\mu \mathrm{m})$ and poor discrimination of plaque subtypes cannot assess the detailed plaque morphology. In the present OCT study, we observed significantly higher prevalence of macrophages in both culprit and nonculprit lesions in PAD patients. Macrophages are key inflammatory cells that are involved at every phase of atherosclerosis, from its genesis to plaque rupture or erosion. Recent studies found that PAD was associated with increased levels of local proinflammatory cytokines, some of which were known to be linked to CAD progression [20-22]. A previous study showed that the severity of coronary atherosclerosis was related to the degree of inflammatory response in the affected PAD limb [23]. This result suggests that not PAD itself, but its systemic inflammatory activity may be associated with more severe CAD. In line with this hypothesis and our results, Fleiner et al. [24] reported that intimal macrophage infiltration of iliac, carotid, and renal arteries was the greatest in patients with cardiovascular events in a postmortem histopathological study. These results are consistent with our knowledge that atherosclerosis is a panvascular process and that inflammation is a key component of atherogenesis $[18,25]$. Inflammation is one of the processes that lead to the formation of lipid core, which contributes to lipid burden in the vascular wall, and thus may explain the greater lipid plaque content and higher prevalence of TCFA in PAD patients in our study [26]. Although difference in the prevalence of TCFA was not statistically significant, TCFA was twice as frequent in patients with PAD compared with that in patients without PAD (32.4 vs. $16.2 \%$ in culprit lesions and 20.0 vs. $11.7 \%$ in nonculprit lesions). Of note, cardiovascular magnetic resonance study reported that patients with more advanced CAD had a higher prevalence of lipidrich necrotic core in their carotid arteries [27]. Combined with our results, these data may imply the coexistence of a higher prevalence of LRP in both coronary arteries and peripheral arteries in patients with concomitant PAD and CAD. Furthermore, several studies reported that patients with an abnormal ankle-brachial index, greater carotid artery intima media thickness, or greater carotid artery stenosis have more complex CAD [28-31]. Moreover, a recent study observed an association of total peripheral score, which evaluated the extent of lower-extremity artery disease, with SYNTAX score [32]. The panvascular inflammatory process observed in our study is consistent with previous findings.

Our matched analysis showed that patients with PAD had a higher prevalence of calcification. This result is in line with previous reports [19]. PAD patients in our study had greater lipid burden and higher prevalence of macrophage accumulation. In more advanced cases of CAD, elevated lipid content and inflammatory mediators induce osteogenic differentiation of vascular smooth muscle cells in the intima. Moreover, patients with PAD typically have reduced physical activity. It has been suggested that sedentary behavior may lead to progression of calcification in the coronary arteries $[18,33]$.

Cholesterol crystals are more frequently found in arteries with greater plaque burden and in those with a higher degree of inflammation [34,35]. This may be a potential explanation for the observed higher prevalence of cholesterol crystals in the lesions of PAD patients.

This study may provide an explanation for higher adverse event rates in patients with concomitant PAD and CAD. First, large lipid pool, TCFA, and macrophages are the major components of vulnerable plaque [36]. Recently, we observed a higher incidence of irregular protrusion after placing stents on an LRP [37]. In case of plaque rupture, large lipid cores may translate into more severe and prolonged thrombotic response [38]. Macrophages, as a component of vulnerable plaque, secrete matrix metalloproteinase, which destabilizes plaque and expresses a tissue factor that is a potent promoter of coagulation [39,40]. Furthermore, increased prevalence of macrophages has been observed in autopsy studies of myocardial infarction patients when compared with that of stable patients [41]. Second, although calcification is not the marker of plaque vulnerability, calcified lesions may influence stent expansion [42,43]. Third, OCT-derived cholesterol crystals may be one of the features of the vulnerable plaque [44]. Several groups reported the association between the presence of cholesterol crystals and acute coronary death $[41,45,46]$. Cholesterol is present in a liquid state in arterial walls. However, factors such as saturation, temperature, pressure, and $\mathrm{pH}$ may individually or in combination trigger the crystallization of the cholesterol within the vessel wall [46]. When cholesterol crystalizes from a liquid state to a solid state, it expands in volume and contributes to the risk for a cardiovascular event [45]. In addition, 
cholesterol crystals induce inflammation and, in consequence, destabilize atherosclerotic plaque [35].

Our study demonstrated greater panvascular vulnerability in patients with combined PAD and CAD. Therefore, this group of patients may have additional benefit from more aggressive risk management. Magnitude of local inflammation may be modulated by high-intensity statin therapy $[22,47]$. A recent study showed that increased cytokine levels lead to platelet hyperactivation [48]. Therefore, potent dual-antiplatelet therapy may have an additional value in this subset of patients.

\section{Limitations}

Several limitations of this study should be noted. First, though data were collected prospectively, the analyses were performed retrospectively. Second, both frequencydomain and time-domain OCTs were used. However, both systems have light sources with the same center wavelength $(1300 \mathrm{~nm})$ and bandwidth, resulting in similar axial resolution $(15 \mu \mathrm{m})$. Therefore, it is unlikely that this difference influenced the results of this study. Third, the number of patients in our study is relatively small. Although we observed differences between the two groups of patients, our observations should be confirmed by larger studies. Fourth, cholesterol crystals described by OCT were not histologically validated. Finally, we did not assess the severity of PAD.

\section{Conclusion}

In the present study, compared with patients with only CAD, patients with concomitant PAD and CAD had greater plaque vulnerability in the coronary arteries, suggesting more generalized inflammatory processes in this group.

\section{Acknowledgements}

The authors thank all the investigators and all supporting staff. Dr. Jang's research was supported by 'Mr. and Mrs. Michael and Kathryn Park' and by 'Mrs. and Mr. Gill and Allan Gray'. Dr. Thomas Zanchin was supported by the Swiss National Science Foundation (grant number 323530_171146). Dr. Tomoyo Sugiyama was supported in part by the grant from Strategic International Research Collaboration Program with Harvard Medical School to Cultivate Professionals with Knowledge and Humanity from Japan Society for the Promotion of Science, and the subvention from Sumitomo Life Welfare and Culture Foundation.

\section{Conflicts of interest}

Ik-Kyung Jang has received educational grants and consulting fees from Abbott. For the remaining authors there are no conflicts of interest.

\section{References}

1 Norgren L, Hiatt WR, Dormandy JA, Nehler MR, Harris KA, Fowkes FGR, et al. Inter-society consensus for the management of peripheral arterial disease. Int Angiol J 2007; 26:81-157.

2 Mozaffarian D, Benjamin EJ, Go AS, Arnett DK, Blaha MJ, Cushman M, et al. Heart disease and stroke statistics - 2015 update: a report from the American Heart Association. Circulation 2015; 131:e29-e322.

3 Fowkes FGR, Low L-P, Tuta S, Kozak J. AGATHA Investigators. Anklebrachial index and extent of atherothrombosis in 8891 patients with or at risk of vascular disease: results of the international AGATHA study. Eur Heart $J$ 2006; 27:1861-1867.

4 Steg PG, Bhatt DL, Wilson PWF, D'Agostino R, Ohman EM, Röther J, et al. One-year cardiovascular event rates in outpatients with atherothrombosis. JAMA 2007; 297:1197-1206.

5 Brevetti G, Giugliano G, Brevetti L, Hiatt WR. Inflammation in peripheral artery disease. Circulation 2010; 122:1862-1875.

6 Singh M, Lennon RJ, Darbar D, Gersh BJ, Holmes DR, Rihal CS. Effect of peripheral arterial disease in patients undergoing percutaneous coronary intervention with intracoronary stents. Mayo Clin Proc 2004; 79:1113-1118.

7 Tendera M, Aboyans V, Bartelink M-L, Baumgartner I, Clément D, Collet J-P, et al. ESC Guidelines on the diagnosis and treatment of peripheral artery diseases. Eur Heart J 2011; 32:2851-2906.

8 Jang I-K, Tearney GJ, MacNeill B, Takano M, Moselewski F, Iftima N, et al. In vivo characterization of coronary atherosclerotic plaque by use of optical coherence tomography. Circulation 2005; 111:1551-1555.

9 Kato K, Yonetsu T, Kim S-J, Xing L, Lee H, McNulty I, et al. Comparison of nonculprit coronary plaque characteristics between patients with and without diabetes: a 3-vessel optical coherence tomography study. JACC Cardiovasc Interv 2012; 5:1150-1158.

10 Nicholls SJ, Tuzcu EM, Crowe T, Sipahi I, Schoenhagen P, Kapadia S, et al. Relationship between cardiovascular risk factors and atherosclerotic disease burden measured by intravascular ultrasound. J Am Coll Cardiol 2006; 47:1967-1975.

11 Cassar A, Poldermans D, Rihal CS, Gersh BJ. The management of combined coronary artery disease and peripheral vascular disease. Eur Heart J 2010; 31:1565-1572.

12 Kato K, Yonetsu T, Jia H, Abtahian F, Vergallo R, Hu S, et al. Nonculprit coronary plaque characteristics of chronic kidney disease. Circ Cardiovasc Imaging 2013; 6:448-456.

13 Kato K, Yonetsu T, Kim S-J, Xing L, Lee H, McNulty I, et al. Nonculprit plaques in patients with acute coronary syndromes have more vulnerable features compared with those with non-acute coronary syndromes: a 3-vessel optical coherence tomography study. Circ Cardiovasc Imaging 2012; 5:433-440.

14 Prati F, Regar E, Mintz GS, Arbustini E, Di Mario C, Jang I-K, et al. Expert review document on methodology, terminology, and clinical applications of optical coherence tomography: physical principles, methodology of image acquisition, and clinical application for assessment of coronary arteries and atherosclerosis. Eur Heart J 2010; 31:401-415.

15 MacNeill BD, Jang I-K, Bouma BE, Iftimia N, Takano M, Yabushita H, et al. Focal and multi-focal plaque macrophage distributions in patients with acute and stable presentations of coronary artery disease. J Am Coll Cardio/ 2004; 44:972-979.

16 Di Vito L, Yoon JH, Kato K, Yonetsu T, Vergallo R, Costa M, et al. Comprehensive overview of definitions for optical coherence tomographybased plaque and stent analyses. Coron Artery Dis 2014; 25:172-185.

17 Yabushita H, Bouma BE, Houser SL, Aretz HT, Jang I-K, Schlendorf KH, et al. Characterization of human atherosclerosis by optical coherence tomography. Circulation 2002; 106:1640-1645.

18 Grenon SM, Vittinghoff E, Owens CD, Conte MS, Whooley M, Cohen BE. Peripheral artery disease and risk of cardiovascular events in patients with coronary artery disease: Insights from the Heart and Soul Study. Vasc Med Lond Engl 2013; 18:176-184.

19 Hussein AA, Uno K, Wolski K, Kapadia S, Schoenhagen P, Tuzcu EM, et al. Peripheral arterial disease and progression of coronary atherosclerosis. $J \mathrm{Am}$ Coll Cardiol 2011; 57:1220-1225.

20 McDermott MM, Guralnik JM, Corsi A, Albay M, Macchi C, Bandinelli S, et al. Patterns of inflammation associated with peripheral arterial disease: the InCHIANTI study. Am Heart J 2005; 150:276-281.

21 Cauley JA, Kassem AM, Lane NE, Thorson S. Prevalent peripheral arterial disease and inflammatory burden. BMC Geriatr 2016; 16:213.

22 Donaldson CW, Schneider DJ, Bertges DJ, Adams JE, Elgharib NZ, Mueller EL, et al. Increased local cytokine production at culprit superficial femoral artery plaques. J Thromb Thrombolysis 2013; 36:293-299.

23 Brevetti G, Piscione F, Schiano V, Galasso G, Scopacasa F, Chiariello M. Concomitant coronary and peripheral arterial disease: relationship between 
the inflammatory status of the affected limb and the severity of coronary artery disease. J Vasc Surg 2009; 49:1465-1471.

24 Fleiner M, Kummer M, Mirlacher M, Sauter G, Cathomas G, Krapf R, et al. Arterial Neovascularization and inflammation in vulnerable patients. Circulation $2004 ; 110: 2843-2850$.

25 Libby P. Inflammation in atherosclerosis. Arterioscler Thromb Vasc Biol 2012; 32:2045-2051.

26 Bentzon JF, Otsuka F, Virmani R, Falk E. Mechanisms of plaque formation and rupture. Circ Res 2014; 114:1852-1866.

27 Underhill HR, Yuan C, Terry JG, Chen H, Espeland MA, Hatsukami TS, et al. Differences in carotid arterial morphology and composition between individuals with and without obstructive coronary artery disease: a cardiovascular magnetic resonance study. J Cardiovasc Magn Reson 2008; 10:31.

28 Sebastianski M, Narasimhan S, Graham MM, Toleva O, Shavadia J, Abualnaja $S$, et al. Usefulness of the ankle-brachial index to predict high coronary SYNTAX scores, myocardium at risk, and incomplete coronary revascularization. Am J Cardiol 2014; 114:1745-1749.

29 Erkan H, Vatan B, Ağaç MT, Korkmaz L, Erkan M, Kırış A, et al. Relationship between SYNTAX score and Trans-Atlantic Inter-Society Consensus II classification in patients undergoing diagnostic angiography. Adv Interv Cardiol 2013; 9:344-347.

30 Ikeda N, Kogame N, lijima R, Nakamura M, Sugi K. Carotid artery intimamedia thickness and plaque score can predict the SYNTAX score. Eur Heart J 2012; 33:113-119.

31 Hur DJ, Kizilgul M, Aung WW, Roussillon KC, Keeley EC. Frequency of coronary artery disease in patients undergoing peripheral artery disease surgery. Am J Cardiol 2012; 110:736-740.

32 Vuruskan E, Saracoglu E, Polat M, Duzen IV. Prediction of coronary artery disease severity in lower extremity artery disease patients: a correlation study of TASC II classification, Syntax and Syntax II scores. Cardiol J 2017. [Epub ahead of print].

33 Delaney JAC, Jensky NE, Criqui MH, Whitt-Glover MC, Lima JAC, Allison MA. The Association between physical activity and both incident coronary artery calcification and ankle brachial index progression: the multiethnic study of atherosclerosis. Atherosclerosis 2013; 230:278-283.

34 Patel R, Janoudi A, Vedre A, Aziz K, Tamhane U, Rubinstein J, et al. Plaque rupture and thrombosis are reduced by lowering cholesterol levels and crystallization with ezetimibe and are correlated with fluorodeoxyglucose positron emission tomography. Arterioscler Thromb Vasc Biol 2011; 31:2007-2014

35 Janoudi A, Shamoun FE, Kalavakunta JK, Abela GS. Cholesterol crystal induced arterial inflammation and destabilization of atherosclerotic plaque. Eur Heart J 2016; 37:1959-1967.
36 Virmani R, Burke AP, Farb A, Kolodgie FD. Pathology of the vulnerable plaque. J Am Coll Cardiol 2006; 47 (Suppl):C13-C18.

37 Bryniarski KL, Tahk S-J, Choi S-Y, Soeda T, Higuma T, Yamamoto E, et al. Clinical, angiographic, IVUS, and OCT predictors for irregular protrusion after coronary stenting. Eurolntervention 2017; 12:e2204-e2211.

38 Madder RD, Husaini M, Davis AT, VanOosterhout S, Harnek J, Götberg M, et al. Detection by near-infrared spectroscopy of large lipid cores at culprit sites in patients with non-ST-segment elevation myocardial infarction and unstable angina. Catheter Cardiovasc Interv 2015; 86:1014-1021.

39 Shah PK, Falk E, Badimon JJ, Fernandez-Ortiz A, Mailhac A, Villareal-Levy G et al. Human monocyte-derived macrophages induce collagen breakdown in fibrous caps of atherosclerotic plaques. Potential role of matrix-degrading metalloproteinases and implications for plaque rupture. Circulation 1995; 92:1565-1569.

40 Zaman AG, Helft G, Worthley SG, Badimon JJ. The role of plaque rupture and thrombosis in coronary artery disease. Atherosclerosis 2000; 149:251-266.

41 Virmani R, Kolodgie FD, Burke AP, Farb A, Schwartz SM. Lessons from sudden coronary death: a comprehensive morphological classification scheme for atherosclerotic lesions. Arterioscler Thromb Vasc Biol 2000; 20:1262-1275.

42 Kobayashi Y, Okura H, Kume T, Yamada R, Kobayashi Y, Fukuhara K, et al. Impact of target lesion coronary calcification on stent expansion. Circ $J$ 2014; 78:2209-2214.

43 Ong DS, Lee JS, Soeda T, Higuma T, Minami Y, Wang Z, et al. Coronary calcification and plaque vulnerability: an optical coherence tomographic study. Circ Cardiovasc Imaging 2016; 9:e003929.

44 Nishimura S, Ehara S, Hasegawa T, Matsumoto K, Yoshikawa J, Shimada K. Cholesterol crystal as a new feature of coronary vulnerable plaques: an optical coherence tomography study. J Cardiol 2017; 69:253-259.

45 Abela GS. Cholesterol crystals piercing the arterial plaque and intima trigger local and systemic inflammation. J Clin Lipidol 2010; 4:156-164.

46 Vedre A, Pathak DR, Crimp M, Lum C, Koochesfahani M, Abela GS. Physical factors that trigger cholesterol crystallization leading to plaque rupture. Atherosclerosis 2009; 203:89-96.

47 Hou J, Xing L, Jia H, Vergallo R, Soeda T, Minami Y, et al. Comparison of intensive versus moderate lipid-lowering therapy on fibrous cap and atheroma volume of coronary lipid-rich plaque using serial optical coherence tomography and intravascular ultrasound imaging. Am J Cardiol 2016; 117:800-806.

48 Bester J, Pretorius E. Effects of IL- $1 \beta$, IL- 6 and IL- 8 on erythrocytes, platelets and clot viscoelasticity. Sci Rep 2016; 6:32188. 